\title{
EchoGéo
}

$8 \mid 2009$

Moyen-Orient : conflits et mobilités dans un espace mondialisé

\section{Le lait local en périphérie de Bamako : une filière en sursis?}

Sophie Molina d'Aranda de Darrax

\section{(2) OpenEdition}

Journals

Édition électronique

URL : https://journals.openedition.org/echogeo/11012

DOI : 10.4000/echogeo. 11012

ISSN : 1963-1197

Éditeur

Pôle de recherche pour l'organisation et la diffusion de l'information géographique (CNRS UMR 8586)

Référence électronique

Sophie Molina d'Aranda de Darrax, «Le lait local en périphérie de Bamako : une filière en sursis ?», EchoGéo [En ligne], 8| 2009, mis en ligne le 19 mars 2009, consulté le 31 juillet 2021. URL : http:// journals.openedition.org/echogeo/11012; DOI : https://doi.org/10.4000/echogeo.11012

Ce document a été généré automatiquement le 31 juillet 2021.

EchoGéo est mis à disposition selon les termes de la licence Creative Commons Attribution - Pas d'Utilisation Commerciale - Pas de Modification 4.0 International (CC BY-NC-ND) 


\title{
Le lait local en périphérie de Bamako : une filière en sursis?
}

\author{
Sophie Molina d'Aranda de Darrax
}

\section{Introduction}

1 La plupart des pays d'Afrique subsaharienne sont dépendants des importations pour leur approvisionnement en produits de première nécessité, alimentaires en particulier. Cette dépendance leur porte fortement préjudice lors de hausses des prix sur les marchés mondiaux d'échanges. Pour le Mali, pays enclavé au cœur de l'Afrique de l'Ouest, le prix des denrées est d'autant plus élevé que le transport est coûteux. Un des moyens de s'émanciper de cette dépendance est le développement de filières de production locales.

2 Des réseaux ont été progressivement mis en place afin d'approvisionner les villes, à partir des ports de la région (Abidjan, Dakar, Conakry, Accra, Lomé, ou encore Cotonou depuis les crises politiques ivoiriennes), mais aussi dans la périphérie de Bamako (Galtier, 2002 ; Raton, 2005 ; Gazull, 2009). Depuis une vingtaine d'années, un système de foires et l'accroissement du nombre de cars rapides permettent de réguler l'approvisionnement de la ville en denrées alimentaires (Raton, thèse en cours).

Etudié dans le cadre de l'Action thématique programmée (ATP) Icare menée par le Centre de Coopération Internationale en Recherche Agronomique pour le Développement (CIRAD), l'approvisionnement des villes en lait est d'autant plus important qu'il s'agit d'une denrée de base et que les villes sont fortement dépendantes des produits laitiers d'importation pour satisfaire la demande de leurs habitants (Pinaud, 2007). Lors d'augmentation du prix du lait importé comme celle de fin 2007 début 2008, avec une augmentation de $30 \%$ du prix du lait en poudre sur le marché mondial, les habitants des villes sont particulièrement touchés. La population bamakoise notamment, puisque Bamako, qui compte aujourd'hui plus d'1,5 millions d'habitants et dont la consommation de lait est en hausse comme dans la plupart des 
villes d'Afrique (Banque Mondiale, 2007), dépend à $95 \%$ des produits d'importation pour satisfaire la demande urbaine (Corniauxet al., art. à paraître).

4 La dépendance vis-à-vis des importations ne cesse de s'accroitre (Pinaud 2007) tandis que, de son côté, l'offre locale de lait semble en proie à un certain nombre de freins. Quoique le prix du lait local soit compétitif et que le marché de consommation du lait augmente à Bamako (Ouologemet al, 2007), la production laitière locale ne progresse pas. Les recherches menées jusqu'à présent par le CIRAD ont permis de mieux cerner les contraintes d'ordre zootechnique, économique et sociologique qui pèsent sur l'élevage en périphérie de Bamako (Poccard Chapuis et al). Elles ont également fait apparaître un besoin d'analyse en termes de contraintes foncières et territoriales pour compléter leurs observations. En effet, outre la faible productivité du cheptel et les difficultés de commercialisation du lait local, l'élevage doit faire face à une contrainte spatiale accrue $\mathrm{du}$ fait de l'expansion urbaine rapide de Bamako. Ce travail se propose de combler en partie ce besoin d'information, en travaillant sur un axe particulier du réseau d'approvisionnement de Bamako en lait : la route de Koulikoro. C'est le principal pourvoyeur de la ville en lait frais, mais c'est aussi un axe privilégié d'expansion du bâti urbain. Quelles sont les dynamiques foncières et territoriales à l'œuvre menaçant le développement, voire la présence de la filière laitière autour de Bamako?

Afin de répondre à cette question, nous commencerons par nous pencher sur les dynamiques spatiales en périphérie urbaine pour comprendre les enjeux de localisation de l'élevage bovin laitier, avant de nous intéresser aux acteurs pratiquant cette activité puis aux perspectives d'avenir auxquelles la filière laitière fait face aujourd'hui.

\section{Méthodologie}

6 L'étude menée a pour objectif de comprendre les dynamiques spatiales, sociales et politiques déterminant la production laitière en périphérie de cette ville. Peu d'études exhaustives étaient préalablement disponibles sur le poids de l'élevage et de la filière lait en périphérie de Bamako. Si les études du CIRAD ont permis d'estimer les quantités de lait produites et apportées à Bamako (environ 5000 litres dont 2000 litres en provenance de l'axe de Koulikoro (Ouologem, 2007), il reste difficile de connaître le nombre d'animaux et les surfaces impliquées dans cette production. Un comptage systématique aurait pris tout le temps du travail de terrain. Cette étude de courte durée - 3 mois - a ainsi davantage été axée sur la compréhension des processus fonciers et territoriaux que sur leur quantification. Le cœur du travail concerne les lieux et la place de l'élevage, ainsi que la compréhension de leur évolution en fonction des enjeux présents dans cet espace de périphérie urbaine. 


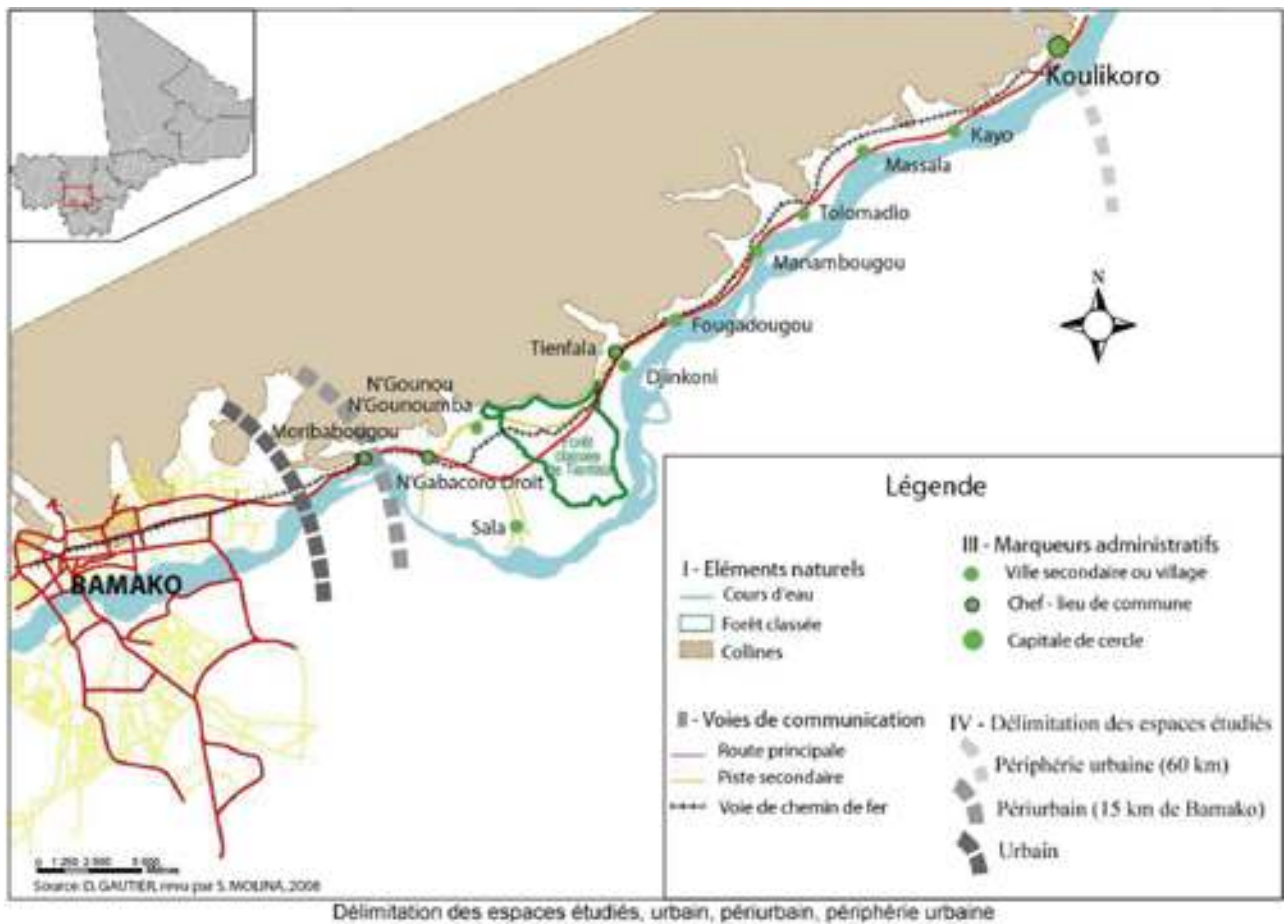

7 Nous avons procédé par administration d'entretiens aux acteurs influant sur les dynamiques de production laitière, propriétaires de troupeaux et propriétaires terriens ainsi que gestionnaires administratifs ou traditionnels des terres étudiées, en recoupant les informations obtenues afin d'avoir le moins de biais subjectifs possibles. Nous avons également parcouru le terrain de recherche afin de mener nos propres investigations spatiales et historiques, pour vérifier les informations orales obtenues et les hypothèses formulées au fur et à mesure de l'avancée de ce travail. Nous avons par ailleurs consulté les registres légaux de propriété des terres et leurs archives, et avons recueilli et analysé les données cartographiques fournies par les organismes administratifs de recensement des terres du district de Bamako et du cercle ${ }^{1}$ de Kati.

\section{Elevage laitier, entre pression foncière et nécessité de proximité au marché}

Une étude du ministère de l'agriculture menée en 2005 indique que la totalité des productions de lait frais à destination de la capitale est produite en périphérie de la ville. "Les systèmes périurbains assurent aujourd'hui la quasi-totalité de l'approvisionnement de la ville de Bamako,[...]la totalité pour le lait frais (400000l/an)[...], et ils s'avèrent donc irremplaçables dans la satisfaction, à des prix abordables, des besoins alimentaires en produits frais » (SOFRECO et HN'D, 2005). Mais ces systèmes périurbains font face à un problème de place du fait de l'expansion de l'espace bâti au détriment des espaces agricoles. Les troupeaux, privés de pâturages et d'accès à l'eau, sont contraints de s'éloigner du lieu de consommation du lait. Cela constitue une réelle entrave à l'approvisionnement de la capitale en produits laitiers. En effet, celui-ci est tributaire des réseaux de transport du lait, qui peinent à s'adapter à l'accroissement des distances entre lieu de production et lieu de distribution. L'éloignement des troupeaux de la ville-marché augmente les coûts 
de transport du lait et remet en question l'aptitude des éleveurs et des collecteurs de lait à porter la production laitière aux points de vente habituels.

\section{Expansion spatiale de Bamako et éloignement progressif des troupeaux}

La spécificité de la situation des productions en périphérie urbaine est qu'elles se situent dans un espace sous influence urbaine. Celle-ci se manifeste par une forte pression foncière, contrariant le développement des activités agricoles. Cette pression foncière trouve son origine d'une part dans l'étalement urbain de Bamako et, corollairement, celui des villages situés en périphérie, et d'autre part dans les mécanismes de spéculation foncière inhérents à l'avancée attendue de la ville.

11 Comme toute capitale africaine, Bamako compte de plus en plus d'habitants, du fait de sa croissance naturelle mais aussi parce qu'en tant que centre urbain et capitale elle continue d'attirer une population rurale nombreuse, migrant à titre saisonnier ou définitif.

Graphique 1- Croissance démographique de Bamako entre 1881 et 2001

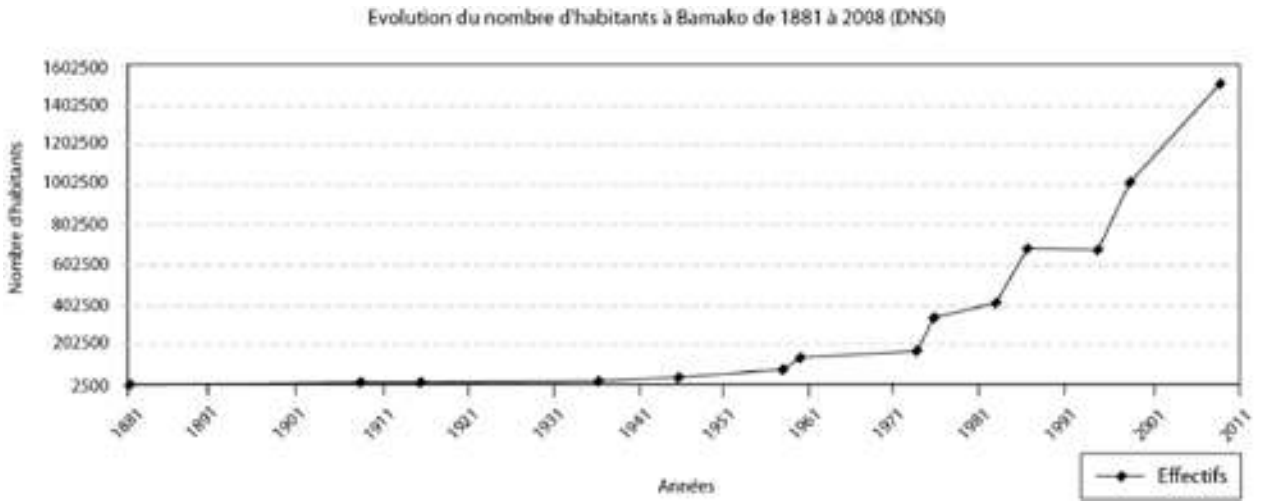

Cet accroissement de la population de la "ville habitée " se traduit par un élargissement spatial de la "ville édifiée » (Choay, 2008). Entre 1960 et 1994, l'emprise au sol de la ville de Bamako a été multipliée par 17 ; elle est passée de 1530 hectares à 26700 hectares (Konate, 2002). 
Carte 2 - Expansion spatiale de Bamako entre 1960 et aujourd'hui

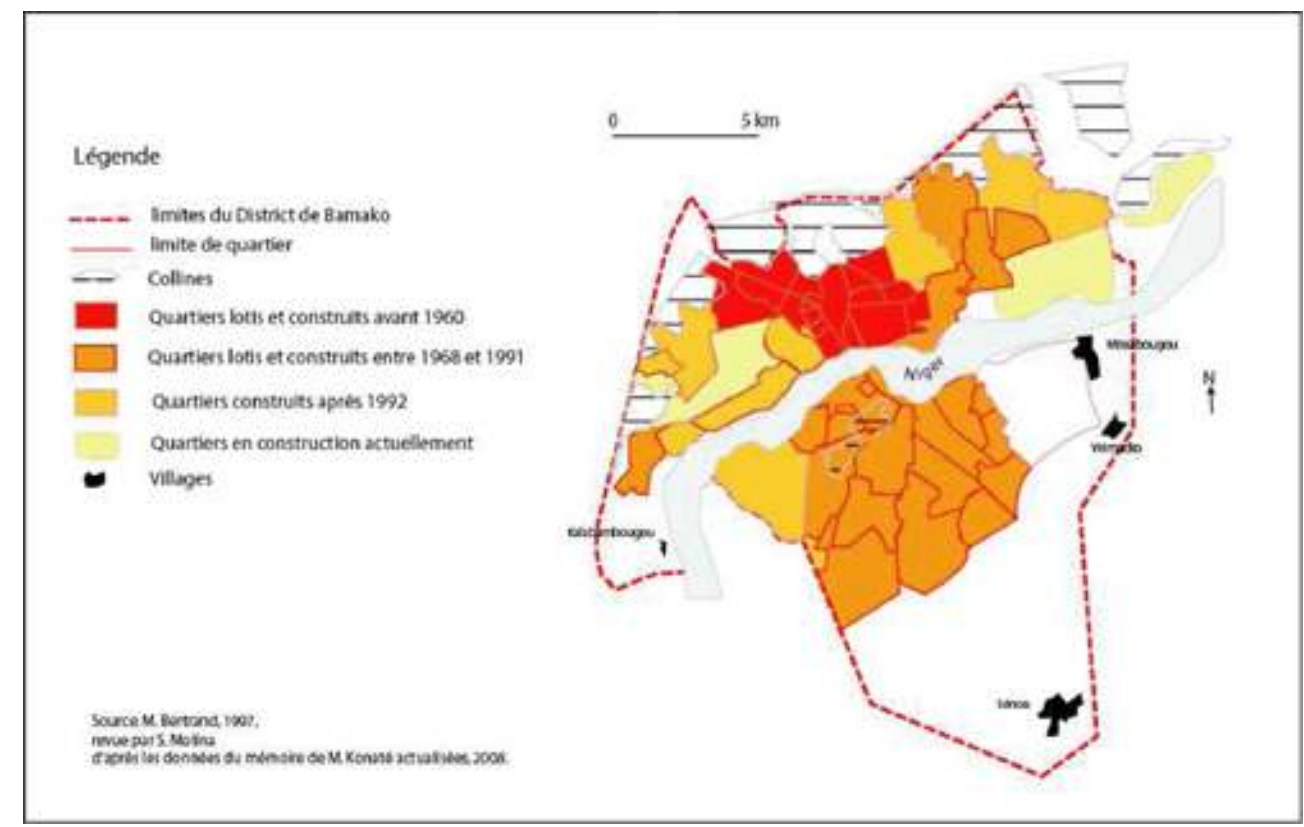

Cet étalement urbain s'explique également par le mode de construction des habitats urbains africains. Comme le note Piermay dans son livre, au sujet de l'Afrique Centrale : «les villes consomment l'espace avec avidité car leurs hôtes conservent une préférence jalouse pour l'habitat horizontal » (Piermay J.L., 1993 : 8).

14 A cette expansion spatiale de Bamako est liée une expansion des villages proches, du fait de l'affluence des populations attirées par la ville de Bamako mais ne trouvant pas où s'y loger à prix accessibles pour elles. L'implantation de ces populations à distance de la ville cible est facilitée par la mise en place d'un réseau de transport efficace entre Bamako et les bourgs ou villes moyennes satellites. C'est le processus que l'on observe à Moribabougou, ville située à une quinzaine de kilomètres de Bamako le long de l'axe de Koulikoro.

L'autre processus contribuant à créer une compétition pour l'utilisation des sols est la spéculation foncière. Elle se manifeste par des clôtures plus ou moins élaborées interdisant l'accès à cet espace approprié, et notamment le passage et le pâturage aux autres éleveurs ou agriculteurs. Ces marqueurs de propriété se trouvent de plus en plus loin de la ville de Bamako. Les propriétaires de ces terrains sont dans leur grande majorité des Bamakois.

Afin de clarifier les spécificités de ces espaces, nous les avons définis et délimités comme suit dans notre travail de master 1, en fonction de l'organisation de l'espace, de l'élevage et du transport des productions laitières (Molina d'Aranda de Darrax s., 2008). :

- urbain, où les sols sont entièrement couverts par l'habitat ;

- périphérie urbaine, où le semi d'habitation est plus lâche quoique continu ;

- périphérie urbaine, où la pression foncière est exclusivement le fait de la spéculation foncière.

17 Du fait de cette pression foncière et de cette compétition pour l'espace, les troupeaux sont peu à peu contraints de s'éloigner des abords de la ville. D'après le chef des Peuls de Moribabougou, arrivé en 1982, c'est peu après que les troupeaux ont commencé à 
affluer vers Moribabougou. Ils arrivaient des quartiers Nord - Est de la ville de Bamako. Dès 1989, certains éleveurs ont pris le parti de les envoyer plus loin à nouveau, face à l'afflux des Bamakois cherchant à se loger à moindre coût que dans la capitale. Les passages des troupeaux vers le fleuve ont été obstrués par les nouveaux murs et les habitants de Moribabougou se sont mis à se plaindre des nuisances olfactives et sonores occasionnées par la présence des animaux, de la poussière soulevée par leur passage et des mouches que les bovins promènent dans leur sillage. Aujourd'hui, il reste encore une bonne cinquantaine d'élevages dans les environs de Moribabougou, mais plusieurs partent chaque année vers Tienfala (village situé à 30 kilomètres de Bamako) et plus loin encore, dans les collines parfois (entretien 26 avril 2008).

A cette contrainte de l'emprise de la ville sur l'espace pastoral s'en ajoute une seconde, liée à la première : celle du transport de la production vers le lieu de vente pour transformation ou consommation.

\section{Transport du lait et déterminants de l'apport en lait}

19 Le drainage des flux de lait produits le long de l'axe Bamako-Koulikoro dépend de plusieurs facteurs. Tout d'abord, le facteur production : pour qu'il soit rentable de transporter son lait vers un lieu de vente, il faut que la production soit suffisante pour que le revenu de la vente couvre les frais de transport et l'inconvénient qu'il y a à se déplacer. Ensuite, le facteur distance entre lieu de production et lieu de vente : il n'est possible et rentable de transporter son lait que si le lieu de vente n'est pas trop éloigné du lieu de production, compte tenu des moyens de transport dont on dispose et du coût de l'essence (650 FCFA le litre en avril 2008). Enfin, le facteur lieu de vente : faut il encore avoir un débouché assuré pour son lait. L'usine MaliLait, par exemple, laiterie située au débouché de cet axe routier Koulikoro-Bamako, ne prend pas le lait en dessous de 30 litres. Et il n'est pas facile de réussir à placer son lait dans les boutiques quand l'apport est irrégulier (variations intersaisons, périodes inter-vêlage). Produire du lait n'est donc pas une garantie d'écoulement, et le fait qu'il existe une dynamique de production laitière en périphérie de Bamako ne garantit pas à la ville son approvisionnement.

La présence des collecteurs privés est alors un point essentiel de l'articulation entre production de lait en périphérie et vente en ville. Ces collecteurs utilisent une moto, un vélo, parfois une charrette à bras. Les collecteurs à vélo vont généralement jusqu'à N'Gabacoro Droit $(15 \mathrm{~km})$, les collecteurs à moto peuvent aller jusqu'à Tienfala $(30 \mathrm{~km})$, où le lait collecté est moins cher, mais le temps de trajet plus long et le coût du transport plus élevé. Ces collecteurs se répartissent les exploitations, voire rivalisent entre eux afin de trouver les exploitations vendant leur lait à bas prix. vente, il existe une séparation des flux de lait au-delà d'une certaine distance à Bamako. 
Figure 1- Schéma: Répartition de la collecte du lait

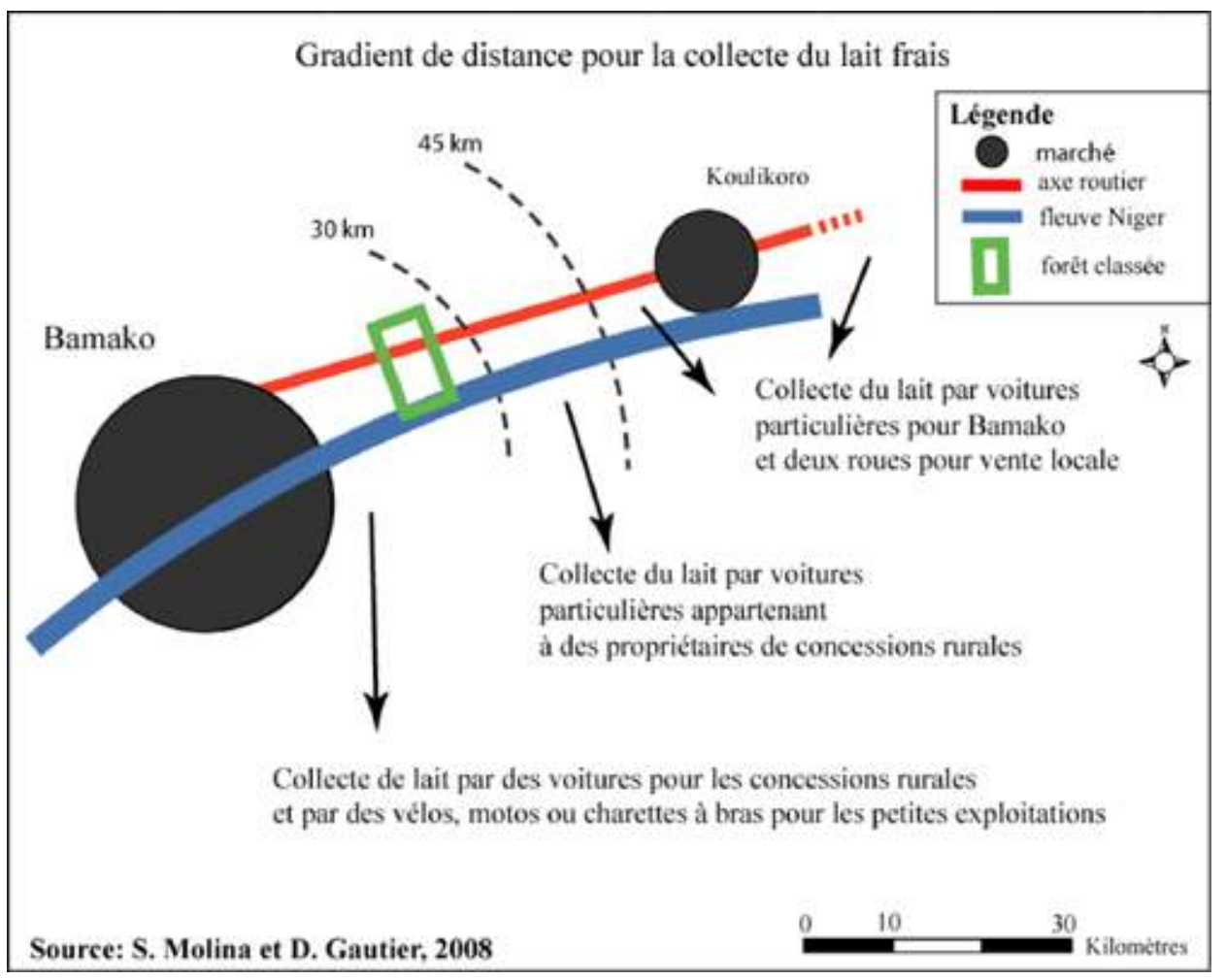

La collecte et la vente du lait le long de l'axe Bamako - Koulikoro se font suivant plusieurs dynamiques. Le lait est parfois vendu sur place par les producteurs. Il peut être vendu de village à village. Il peut enfin être vendu en ville, chez les producteurs directement aux consommateurs, dans des boutiques et boulangeries ou dans une laiterie industrielle.

Il existe deux villes importantes le long de l'axe étudié : Bamako et Koulikoro. Du fait de l'attraction particulièrement forte exercée par Bamako, certains grands producteurs expédient leur lait produit au-delà de Koulikoro à l'usine MaliLait de Bamako. Les petits producteurs situés à proximité de Koulikoro et dont la production ne permet pas de déplacements de cette envergure vendent leur lait à Koulikoro. Si ces petits producteurs se situent dans un rayon de distance inférieur à 30 kilomètres de Bamako, leur production peut être achetée par les collecteurs privés qui se chargent de l'acheminer vers Bamako et de l'y vendre (cf. carte de répartition des flux de lait le long de l'axe routier).

Le flux de lait quotidien vers Bamako est donc déterminé par la quantité produite et les possibilités d'expédition vers Bamako, via des moyens de transport propres au producteur ou via l'intermédiaire des collecteurs. La distance du lieu de production à la ville-marché est une des clefs déterminant le coût du transport. L'éloignement progressif des troupeaux du fait de l'expansion urbaine se présente donc comme une contrainte forte pour l'approvisionnement de Bamako en lait frais. Certains éleveurs ont déjà envoyé leurs troupeaux dans les collines depuis 4-5 ans et ne collectent plus quotidiennement leur lait, dont le transport vers la ville n'est plus rentable.

Après nous être intéressés aux conditions de productions et d'approvisionnement de la ville de Bamako, nous nous intéresserons à présent aux stratégies d'élevage. 


\section{Qui tient la terre tient la production laitière}

Les moyens de continuer à avoir une activité rentable de production de lait à proximité de Bamako sont désormais d'avoir un accès sécurisé au fleuve pour abreuver les animaux et à des pâturages (dont une partie est constituée de champs dont les résidus sont broutés), ce qui signifie en clair avoir une propriété foncière, et de disposer de suffisamment de moyens financiers pour se fournir en aliment de bétail pour alimenter les animaux en complément de pâturages de moins en moins accessibles. Il faut également produire suffisamment de lait et, pour cela, utiliser toutes les techniques d'amélioration de la production à disposition, soit le métissage et la complémentation alimentaire.

\section{Avantages stratégiques de la situation de l'espace étudié}

L'avantage de cet axe Bamako - Koulikoro est qu'il dispose d'atouts naturels, le fleuve notamment, et de la présence d'une route à proximité du fleuve. Cette situation est stratégique pour des activités productives destinées à une commercialisation urbaine. La proximité d'eau facile d'accès est un impératif si l'on souhaite développer un élevage bovin, à la fois pour l'abreuvement des bêtes, mais aussi pour la production de compléments alimentaires. La proximité de la route est, elle, intéressante pour envoyer aisément ses productions vers la ville en grande quantité sans que les coûts de transport soient trop élevés.

Un autre avantage non négligeable est la proximité de la capitale. C'est à Bamako que se trouvent les centres de recherche, notamment le ranch de Sotuba, où sont effectués les travaux d'amélioration de la productivité du cheptel local. C'est ici qu'ont été menées les expériences de métissage et d'insémination artificielles, et c'est à proximité de la capitale que la majorité des inséminations artificielles et des métissages par monte naturelle ont été subventionnés et répandus. Soixante pour cent des inséminations artificielles qui ont été faites à proximité de Bamako l'ont été le long de l'axe BamakoKoulikoro, et c'est le long de cet axe que l'on trouve le plus grand nombre d'animaux métissés, les plus productifs. C'est dans le ranch de Sotuba également qu'ont été effectuées les recherches concernant l'amélioration de la ration alimentaire des animaux et que ces techniques de complémentation alimentaire ont été vulgarisées. Il est donc plus aisé pour les propriétaires de troupeau de la périphérie de Bamako d'être tenus au courant des progrès dans la zootechnie que pour les éleveurs d'autres régions du Mali, et l'accès aux méthodes modernes de production et d'amélioration de la production y est plus facile.

\section{Les propriétaires terriens, typologie et propriété idéale}

On ne peut étudier les différents types d'éleveurs et comprendre les différentes stratégies d'élevage sans s'intéresser au préalable à l'appropriation des sols de la périphérie urbaine.

Traditionnellement, au Mali, l'utilisation des terres est régie par le droit d'usage. «Entre les hommes et la terre n'existent que des droits d'usage et de culture " (" Les Etats Généraux du Foncier au Mali ", 2005). La terre peut appartenir à une collectivité mais pas à un individu, c'est un bien inaliénable. Le chef de village répartit les terres du village entre 
les familles afin qu'elles puissent cultiver selon leurs besoins. Cependant, sous la colonisation, on a cherché à remplacer le droit coutumier oral par un droit moderne écrit, système fondé sur le régime de l'immatriculation des terres allouées, privatisées et inscrites sur le livret foncier.

La privatisation des terres est l'acquisition de terres rurales en périphérie urbaine, appelées "concessions rurales », par des particuliers. Il s'agit de propriétés foncières situées en zone rurale, cédées à un individu privé par l'Etat du Mali. Le droit à la terre traditionnel persiste, mais il est possible aux autorités traditionnelles (le chef du village) et administratives de céder ou de vendre des portions de terre aux particuliers qui en font la demande, d'après le Code Domanial et Foncier du Mali (1986).

Plusieurs vagues d'accès à la terre se sont succédées, selon les différents régimes et dirigeants au pouvoir.

Figure 2 - Historique de l'appropriation du sol le long de l'axe Bamako-Koulikoro

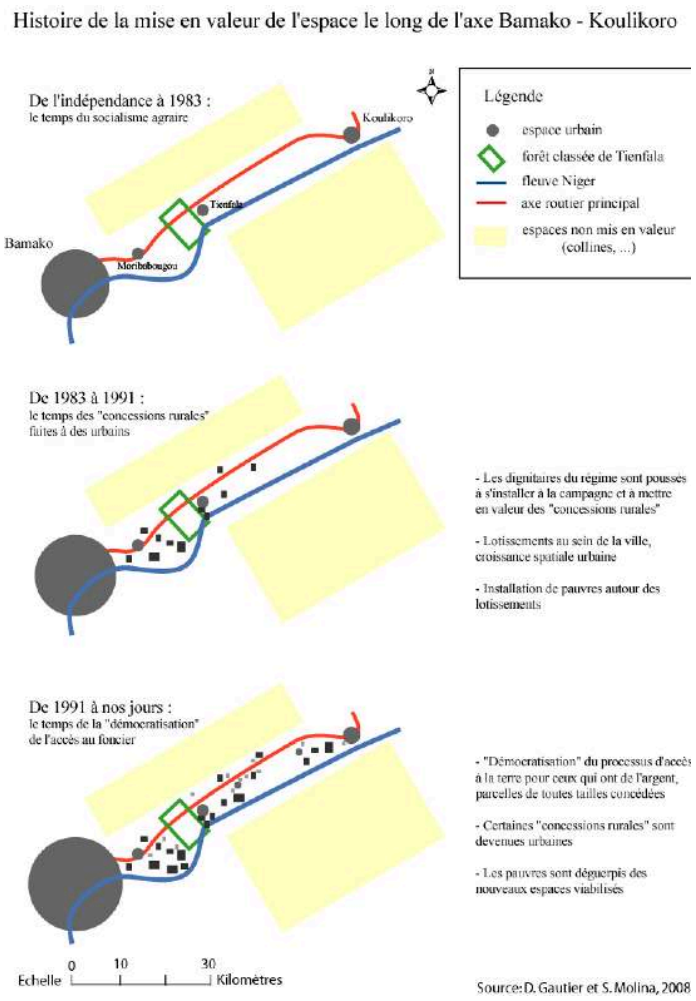

Entre l'indépendance et 1983, l'accès à la terre était malaisé, non garanti par l'Etat, qui pouvait exproprier tout occupant. Les parcelles qui ont été concédées l'ont principalement été à des élites du régime, qui ont acquis de grandes parcelles en bordure de fleuve et à proximité de la ville. Le premier Code Domanial et Foncier du Mali est écrit en 1982 et mis en application en 1983 ; l'accès à la terre est assoupli. Les élites urbaines peuvent à leur tour accéder à la propriété d'une parcelle de terre à proximité de la capitale. Depuis 1991, les procédures d'accès à la terre sont simplifiées : l'immatriculation d'une terre de petite dimension (jusqu'à 2,5 hectares) ne demande plus de passer devant le commandant de cercle mais devant un représentant du gouvernement en mairie par exemple, ce qui facilite les démarches administratives. Toutefois, le coût d'accès à la propriété privé reste élevé et réservé à une frange aisée 
de la population bamakoise. Cette histoire foncière a un impact important sur les usages du sol en périphérie de Bamako et notamment sur les pratiques d'élevage laitier.

Cette revue de l'histoire foncière nous permet à présent d'appréhender la diversité des types d'élevage pratiqués le long de l'axe routier étudié et les différentes stratégies de productions et vente du lait :

- les paysans, disposant d'une ou deux vaches principalement dédiées aux labours et dont la production de lait est une sorte d'effet secondaire bénéfique. Ils consomment eux-mêmes leur lait ou le vendent sur place. Les animaux sont de race locale et ne sont pas complémentés.

- les Peuls migrants ayant reformé un troupeau. consomment eux-mêmes leur lait et vendent le surplus dans les villages proches. S'ils sont situés à moins de 30 kilomètres de Bamako, il est fréquent qu'ils vendent leur surplus à des collecteurs privés. La croissance urbaine est problématique pour leur activité car les espaces de pâturage se réduisent et le fleuve est de moins en moins accessible. Ils sont plusieurs à avoir déjà éloigné leurs troupeaux et à prévoir de l'éloigner encore plus les années à venir.

- les propriétaires de parcs privés situés en périphérie de ville louent un espace de leur parc aux propriétaires de bovins qui ne disposent pas de concessions rurales. Le lait est revendu à un collecteur et l'argent reçu permet au propriétaire bovin de payer le propriétaire du parc, d'entretenir le troupeau et, parfois même, de faire des bénéfices. Ces troupeaux paissent sur les terrains vagues et les champs d'ordures à proximité de Bamako. Ils sont condamnés à plus ou moins long terme à s'éloigner de la ville, lorsque les terrains vagues seront à leur tour couverts d'habitats.

Pour les paysans, peuls et parcs privés, l'espace se ferme. Il est de plus en plus difficile de maintenir les troupeaux à proximité de la ville.

Deux autres catégories font face à d'autres problématiques de production laitières que celles rencontrées par les trois premier types d'élevage périurbains exposés : les propriétaires de concessions rurales de la première vague d'appropriation foncière et ceux nouvellement arrivés.

Les premiers ont décidé d'élever un troupeau dans un but lucratif ou récréatif ou les deux. La productivité du troupeau est élevée et le troupeau est soigné. Leurs animaux sont généralement métissés avec des races européennes, Montbéliard ou Holstein, et les bovins sont complémentés avec une alimentation de qualité (tourteau de coton). Ils produisent entre 40 et 150 voire 200 litres de lait par jour qu'ils apportent en ville. D'après nos relevés, il semble qu'ils produisent entre un tiers et la moitié de la production laitière de l'axe, soit un peu moins de mille litres de lait par jour. Etant propriétaires fonciers ( 2 à 50 hectares), possédant les titres fonciers, ils ne craignent pas l'avancée de la ville. Les moyens financiers dont ils disposent leurs permettent d'utiliser des moyens de transport du lait privés et leurs exploitations semblent rentables. Celles qui ne le sont pas et dont le troupeau est conservé pour le plaisir de posséder des bovins portent tout de même le surplus de lait à MaliLait ou dans une boulangerie de la ville de Bamako. Pour la majorité d'entre eux cependant, le plaisir de la possession de troupeaux s'allie à la rentabilité tirée de l'élevage de ces animaux.

Les seconds ne sont pas encore au fait des techniques de production améliorées et disposent de moyens financiers moins important que les premiers cités, mais ils possèdent tout de même des troupeaux productifs. Ils apportent eux-mêmes le lait dans les lieux de vente urbains ou revendent leur production à un collecteur privé. Ceux-là ont acquis une parcelle de terre également. Cependant, elles sont moins étendues que 
celles des propriétaires cités plus hauts et leur pérennité dépendra de l'avancée de la ville et des moyens financiers dont ils disposent pour entretenir leur troupeau (augmentation de la productivité de leurs vaches, pas toutes métissées, et achat de compléments alimentaires).

Pour ces propriétaires terriens, la possession de titres fonciers garantit la conservation de leurs terrains malgré l'avancée de la ville. Ils peuvent ainsi garder leur troupeau à proximité de Bamako. L'avancée de la ville restreignant toutefois les espaces de pâture, ils devront intensifier la complémentation alimentaire de leurs animaux s'ils souhaitent maintenir leur productivité. Sur le long terme, le maintien de la production en périphérie urbaine passe par son intensification.

\section{Perspectives de production laitière : sur quel front lutter?}

40 Outre les problèmes de pression foncière rencontrés par la plupart des éleveurs, la production laitière rencontre des obstacles internes aux exploitations (difficultés de succession) ou externes (difficulté d'organisation d'une collecte commune du lait) qui entravent son développement actuel et futur le long de cet axe.

\section{Successions et reprises d'exploitations en suspens}

Les exploitations les plus productives aujourd'hui sont les exploitations des dignitaires de la seconde république. Au sein de ces exploitations, le principal obstacle au maintien et au développement de la production laitière est la question de la reprise de ces exploitations par les descendants des propriétaires.

42 Après la chute du gouvernement de Moussa Traoré (1991) qui, prônant un retour à la terre, avait relancé une dynamique de production laitière en périphérie de Bamako et octroyé des terres à ses dignitaires afin qu'ils pratiquent l'élevage, de nombreuses exploitations non rentables ont disparu.

43 Aujourd'hui, les premiers exploitants ont à trouver des successeurs pour reprendre leur exploitation, sous peine qu'elle disparaisse. C'est le cas de celle de B.G., propriétaire d'une parcelle de plusieurs hectares près de Massala. Il possédait un troupeau de bovins produisant entre 30 et 70 litres de lait par jour selon la saison. Lorsqu'il est mort, seul un de ses enfants a souhaité reprendre l'exploitation. La majeure partie du troupeau a été vendue, l'exploitation démantelée; le fils ayant repris l'exploitation doit à présent recommencer progressivement à réorganiser l'exploitation et le troupeau.

Ce cas de figure, relativement fréquent puisque nous avons repéré quatre propriétés sur la trentaine étudiée dont les descendants n'avaient pas repris l'exploitation, représente une déperdition productive pour Bamako. Il tient à la fois au changement de paradigme sociétal au Mali (politique de retour à la terre dépassée) et au fait que la pratique de l'élevage et de la vente du lait n'est plus aussi aisée aujourd'hui que lorsque les premiers dignitaires ont commencé à se lancer dans la production laitière. Ils étaient soutenus par la politique socialiste menée par le gouvernement : accès aisé aux ressources alimentaires (tourteau de coton, issu des cultures de la CMDT transformées par l'huilerie d'Etat, Huicoma) et aux inséminations proposées par le ranch de Sotuba, assurance d'écouler le lait produit à un bon prix dans la laiterie d'Etat (Union Laitière 
de Bamako, actuelle MaliLait),... soit des avantages auxquels leurs descendants ne peuvent plus prétendre, étant donnés le changement de gouvernement et d'orientation économique (socialisme d'Etat vs libéralisme) et la dynamique de privatisation de l'industrie impliquée dans la production laitière (MaliLait, 1994, Huicoma, 2005, CMDT, en cours).

\section{Compléments alimentaires et rentabilité}

Si l'augmentation du prix de vente du lait sur le marché mondial se présente comme une aubaine pour les producteurs laitiers locaux, qui peuvent également écouler le leur à prix plus élevé, l'augmentation des prix d'autres produits leur porte en revanche fortement préjudice. C'est le cas de l'augmentation du prix de l'aliment bétail, qui a été multiplié par deux en 2007-2008, la tonne étant passée de 90000 FCFA à 170000 FCFA.

L'aliment de bétail est un élément primordial pour le maintien de la productivité des vaches laitières en périphérie de Bamako. Tout d'abord parce que les vaches métissées ont besoin de ce complément alimentaire. Ensuite parce que les vaches locales sont nettement plus productives lorsqu'elles reçoivent une ration de complément alimentaire régulièrement. Enfin, parce qu'avec le manque de pâturages en périphérie urbaine l'aliment de bétail permet d'entretenir la productivité et la fertilité des troupeaux qui, sans cela, péricliteraient.

L'augmentation du prix du tourteau, principal aliment du bétail, amène les éleveurs soit à réduire leur production laitière en diminuant les rations octroyées aux animaux, soit à changer d'aliment de bétail, soit à acheter l'aliment au prix double, ce qui diminue alors fortement la rentabilité de l'exploitation.

Des projets de développement d'autres types de compléments alimentaires végétaux sont en cours, mais ils ne sont pas encore opérationnels. Certains éleveurs, peu nombreux, tirent leur épingle du jeu en donnant à leurs animaux du maïs ou en créant leur propre complémentation. Le gouvernement a toutefois engagé des efforts dans ce sens, faisant paraitre dans l'Essor (quotidien local) une recette de complémentation bétail pour inciter les éleveurs à diversifier la provenance et la composition des compléments habituellement utilisés.

\section{Mobilisation collective et coopération entre éleveurs}

Une autre entrave réside dans l'incapacité dont font preuve les éleveurs le long de cet axe à s'accorder entre eux afin de s'associer pour récolter leur lait et l'expédier vers Bamako en commun. Plusieurs tentatives d'organisation ont vu le jour mais elles ont toutes avorté.

La justification apportée par les éleveurs était unanime : ils ont tous essayé de former la leur, mais la mauvaise volonté des autres a chaque fois fait échouer le projet. Par exemple, il y eut un projet ayant pour objectif de concurrencer MaliLait, dont le nouveau directeur, après la privatisation, avait refusé de partager la gestion avec les éleveurs qu'il considérait comme peu fiables. Ce projet avait pour objectif la création d'une laiterie, la Solaima, en partenariat avec la région Franche Comté (1997). Ce projet n'a jamais vu le jour et les crédits avancés par les éleveurs et la région française ont disparu. De même, Monsieur O.F. avait eu un temps le projet de pasteuriser le lait produit le long de l'axe dans son exploitation (2000). Mais s'étant accordé avec les 
éleveurs le long de l'axe, il a cependant remarqué rapidement que certains ajoutaient de l'eau ou apportaient un lait avarié. Le projet a tourné court.

Il a existé tout de même certaines petites coopératives et associations d'éleveurs dans les villages aux environs de Bamako : Sala, ou encore Moribabougou avaient la leur. Elles permettaient d'acheter des médicaments et de l'aliment bétail en gros, qui étaient ensuite revendus à prix relativement intéressants pour les éleveurs participants. Cependant, depuis l'augmentation des prix du tourteau, le mot d'ordre est, comme l'explique un éleveur, «chacun pour soi » (H.D.). Ces coopératives ne fonctionnent plus depuis deux ans et ce constat est identique dans plusieurs villages enquêtés.

\section{Compétition pour le sol et manque de soutien de l'administration}

La dernière entrave au développement de l'élevage bovin laitier le long de cet axe correspond au différend opposant les premiers occupants du sol, villageois, et les propriétaires terriens.

Carte 3 - Sala, village enkysté dans les concessions rurales

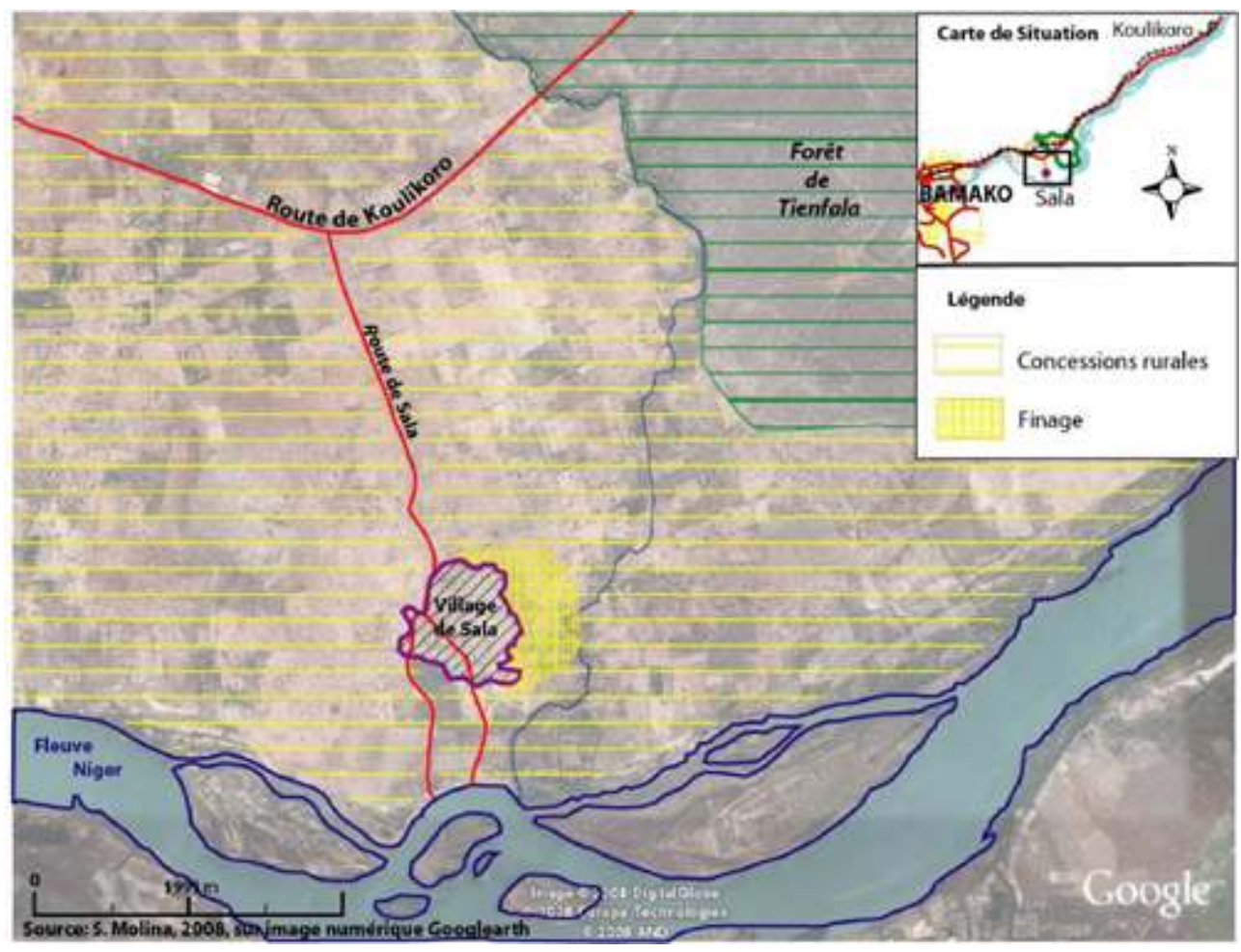

Si les villageois ont cédé une bonne partie de leurs terres, c'est parce qu'elles n'étaient pas utilisées. Pas nécessairement conscients des implications temporelles et spatiales de la vente de leurs sols, certains chefs ont cédé tout l'espace environnant le village, qui se trouve aujourd'hui cerné par les propriétés bamakoises. Réalisée à partir d'une image Googlearth et des enquêtes menées sur place, la carte suivante permet de prendre la mesure de ce mouvement d'appropriation des terres. On peut voir le village de Sala, qui ne dispose plus que d'un finage très réduit, cerné par la mosaïque des parcelles de propriétaires bamakois. Il n'y a plus suffisamment de place pour même autoriser de nouvelles familles à s'installer. Les villageois cultivent les champs que les Bamakois n'ont pas encore clôturés ou doivent aller dans les collines. Pour pouvoir 
implanter de nouvelles habitations villageoises, il faudrait racheter les terres cédées gratuitement aux Bamakois. Or, la prise du sol a tellement augmenté ces dernières années que cela n'est pas envisageable, et les instances administratives ne peuvent exproprier aisément un propriétaire de concession rurale. Cette situation est à l'origine d'un conflit latent entre concessionnaires et villageois, qui n'ont plus ni champs à cultiver ni pâtures ou faire paître leurs animaux.

De même, lors des opérations de lotissements en périphérie de Bamako, la problématique de l'élevage n'est pas prise en compte par les instances de sélection des plans. Les rues sont tracées trop étroites pour que les troupeaux puissent circuler à Moribabougou par exemple, ce qui a forcé les propriétaires à éloigner leurs troupeaux, puisqu'ils n'avaient plus ni pâturages ni accès au fleuve pour leurs animaux à la suite de ces opérations. L'élevage n'est ainsi absolument pas privilégié ou protégé par les institutions, n'étant pas considéré comme une priorité.

\section{Conclusion}

La filière laitière en périphérie de Bamako doit donc lutter sur plusieurs fronts territoriaux pour se maintenir et se développer. En premier lieu, elle doit s'adapter au processus d'étalement spatial de la capitale et des villages environnants, qui force les troupeaux à s'éloigner et rend le transport du lait vers les lieux de vente en ville plus difficile. L'incapacité des acteurs à s'organiser pour réduire les coûts de production et de transport du lait vers Bamako du fait de la mauvaise volonté des éleveurs représente, sinon une menace, du moins une entrave au développement de la filière locale. Le dernier obstacle territorial est le manque de soutien de l'administration et l'absence de politiques publiques favorisant le développement de l'élevage en périphérie urbaine.

A la fois bénéficiaire et victime des différents régimes politiques et mouvements fonciers qui se sont succédé, la production laitière le long de l'axe Bamako-Koulikoro souffre aujourd'hui principalement d'un manque de coordination des acteurs concernant l'aménagement $\mathrm{du}$ territoire (administration - villageois concessionnaires) et l'organisation interne de la filière (producteurs - collecteurs centres de réception et transformation du lait).

57 Afin de soutenir la production laitière pour l'approvisionnement de la ville de Bamako en lait frais, il pourrait être judicieux de mettre en place une collecte commune du lait et d'assurer des débouchés à la production locale, actuellement remplacée par du lait en poudre dans les laiteries de Bamako. Cette mesure porterait toutefois préjudice aux collecteurs privés. Il convient donc de chercher un moyen d'organiser cette collecte de façon à ne pas pénaliser l'activité économique générée le long de l'axe par la présence de cette production laitière.

Il faudrait également prendre en compte l'impératif de la complémentation alimentaire, former les éleveurs pour leur enseigner les différentes recettes de fabrication de compléments alimentaires et accélérer la mise au point et l'implantation des herbes permettant de compenser le manque de tourteau afin que tous y aient accès, quitte à redynamiser les coopératives qui fonctionnaient dans les villages avant la hausse du cours du tourteau de coton. 

permettant de préserver des espaces de pâture et des accès au fleuve pour que les animaux puissent s'abreuver, créer des plans d'aménagement communaux associant villageois et privés afin qu'ils puissent s'entendre sur une répartition de l'espace et des pratiques nuisant le moins possible aux deux parties.

60

observations que nous avons menées et nos recommandations sont toutefois rattachées au contexte précis de la ville de Bamako et, plus particulièrement, à l'axe que nous avons étudié. Le long d'autres axes de production laitière en périphérie de Bamako existent par exemple déjà des points de collecte du lait et une organisation commune du transport vers Bamako. Nos observations ne s'appliqueraient donc pas intégralement à ces espaces, quoique l'expansion de Bamako représente sans doute également un facteur de contrainte non négligeable pour les éleveurs. D'autre part, les enjeux spatiaux et les solutions à appliquer diffèrent nécessairement en périphérie de capitale et en périphérie de ville moyenne. Aussi, afin d'élargir la réflexion concernant le développement des filières de production laitière en périphérie urbaine en Afrique de l'Ouest et de pouvoir faire des recommandations adaptées, il faudrait pouvoir bénéficier d'autres études permettant de prendre en compte la diversité des enjeux spatiaux en fonction des spécificités des villes et des situations locales des élevages périurbains.

\section{BIBLIOGRAPHIE}

Banque Mondiale, 2007, Rapport 2008 sur le développement dans le monde, l'Agriculture au service du développement, The International bank for reconstruction and development, Washington, $27 \mathrm{p}$. choay $\mathrm{F} ., 2008$, Une définition de la ville, http://www.aroots.org/Une-definition-dela-ville.html(mai 2008)

Corniaux Ch., Bonfoh B., Diallo A., Poccard-Chappuis R., Vias G., Réseaux de collecte et de distribution du lait dans les villes d'Afrique soudano-sahéliennes, art. à paraître, $24 \mathrm{p}$.

République du Mali, 2005, « les Etats Généraux du Foncier au Mali », document annexe au Code Domanial et Foncier du 12 juillet 1986 (Général Moussa Traoré), imprimeries du Mali, loi nº 86-91/ANRM portant Code Domanial et Foncier.

Galtier F., 2002, Information, institutions et efficacité des marchés : l'analyse de trois filières céréalières d'Afrique de l'ouest comme des « systèmes de communication ». Thèse de doctorat ENSAM, $519 \mathrm{p}$.

+ annexes.

Gazull L., 2009, Le bassin d'approvisionnement en bois - énergie de Bamako - une approche par un modèle d'interaction spatiale, thèse, Université Paris-Diderot, Dir. : GRASLAND c.

Konate M., 2002, La dynamique de l'espace urbain à Bamako: Réglementation foncière et morphologie des quartiers de Faladie-Sema et de Niamakoro, mémoire de DEA en Géographie urbaine, Université de Bamako. 
Molina d'Aranda de Darrax S., 2008, Organisation de la production laitière en périphérie urbaine en Afrique de l'Ouest, le cas de la route de Koulikoro en périphérie de Bamako, Mali, mémoire de Master 1, Université Paris I Panthéon-Sorbonne, Dir. : Gautier D., Chaléard J.L., Ninot O., 140 p.

Ouologem B. (chef de projet), 2007, Recherche de mode de gestion du troupeau pour une exploitation économique durable des bovins laitiers dans les zones périurbaines du Mali : production, commercialisation et consommation de produits laitiers en zones périurbaines du Mali, Résultats de Recherche 2003-2007.

Pinaud S., 2007, Etude du réseau de distribution des produits laitiers à base de poudre de lait à Bamako, mémoire de Master 2, Université Paris x Nanterre, Dir. : CORNIAUD et VATIN, 105 p.

Piermay J.L., 1993, Citadins et quête du sol dans les villes d'Afrique Centrale, éd. l'Harmattan, coll. Villes et Entreprises, Paris, 579 p.

Poccard-Chappuis R., Corniaux Ch., Coulibaly D., Dynamiques de structuration des bassins laitiers : comparaison entre l'Amazonie Brésilienne et le Mali, art. à paraître, 43 p.

Raton G., 2005 - en cours, Dynamiques périurbaines et approvisionnement de Bamako en produits forestiers, Thèse en cours, Université Paris I Panthéon-Sorbonne, Dir. : Chaléard J.L.

République du Mali, 1987, Code Domanial et Foncier du 12 juillet 1986 (Général Moussa Traoré), imprimeries du Mali, loi n 86-91/AN-RM portant Code Domanial et Foncier.

République du Mali, 2000, Code Domanial et Foncier, ordonnance $n^{\circ}$ 00-027/P-RM du 22 mars 2000, et Lois et Décrets d'Application du Code Domanial et Foncier, Ministère des Domaines de l'Etat et des Affaires Foncières. (Président Alpha Konaté)

République du Mali, 2006, Recueil des textes des Domaines et du Cadastre, ordonnances, lois et décrets. SOFRECO (France) et HN'D (Mali), 2005, Programme d'appui à l'agriculture urbaine et périurbaine du Mali, étude de faisabilité, Rapport définitif, ministère du développement rural, Mali, Bamako.

Staatz J. et N. Dembele,1989, “Cereals market liberalization in Mali”, World Development, 17 (5), p. 7013-718.

\section{NOTES}

1. " district ", " cercle " : entités administratives s'apparentant par leur organisation à nos départements et régions français.

\section{RÉSUMÉS}

$95 \%$ de la consommation de lait de la population bamakoise est assurée par les importations. Pourtant, le Mali est l'un des pays d'Afrique de l'Ouest disposant du cheptel laitier le plus conséquent. L'objet de cette étude est d'interroger les pratiques de production laitière en périphérie de Bamako et leurs déterminants, afin de comprendre pour quelles raisons la production laitière et la part de lait local dans la consommation de la ville n'augmentent pas malgré la demande urbaine croissante, et d'envisager des solutions pour y remédier. Pour ce faire, nous analyserons les paramètres physiques, territoriaux et techniques qui entravent la 
production laitière en périphérie de Bamako et conditionnent la quantité de lait local disponible, sur la base d'études de terrain et d'interviews d'acteurs réalisées entre février et mai 2008.

95\% of Bamako's dairy products consumption is ensured through milk powder importations, while Mali is one of the most important dairy cattle countries in West Africa. This study aims at analyzing milk production practices and determining elements to understand why milk production around Bamako doesn't raise despite that consumption is growing fast, and propose solutions to improve that situation. In order to answer that question, we'll study physical, geographical and technical aspects of milk production around Bamako through interviews and field work realized between February and May, 2008.

INDEX

Mots-clés : appropriation foncière, périurbanisation, production laitière, élevage

Keywords : suburbanization, land property, dairy-farming

\section{AUTEUR}

\section{SOPHIE MOLINA D'ARANDA DE DARRAX}

Sophie Molina d'Aranda de Darrax (sophie.molinadarandadedarrax@essec.fr) est actuellement en MBA à l'ESSEC 